7th International Workshop on Astronomy and

Relativistic Astrophysics (IWARA 2016)

International Journal of Modern Physics: Conference Series

Vol. 45 (2017) 1760053 (8 pages)

(C) The Author(s)

DOI: $10.1142 / \mathrm{S} 2010194517600539$

\title{
Rotational Energy Transfer Non-Reactive Hydrogen-Hydrogen Collisions at Low Temperatures of Astrophysical Interest
}

\author{
Renat A. Sultanov* \\ Business Computing Research Laboratory (BCRL) \\ St. Cloud State University \\ 367B Centennial Hall, 720 Fourth Avenue South \\ St. Cloud, Minnesota 56301, USA \\ rasultanov@stcloudstate.edu \\ Dennis Guster \\ Department of Information Systems and BCRL \\ St. Cloud State University \\ 367C Centennial Hall, 720 Fourth Avenue South \\ St. Cloud, Minnesota 56301, USA \\ dcguster@stcloudstate.edu
}

Published 15 August 2017

\begin{abstract}
We perform a pure quantum-mechanical calculation for the non-reactive scattering in atomic and molecular hydrogen-hydrogen collisions, i.e. $\mathrm{H}_{2}+\mathrm{H}_{2}$ and $\mathrm{H}+\mathrm{H}_{2}$. Different potential energy surfaces (PESs) for the $\mathrm{H}_{3}$ and $\mathrm{H}_{4}$ atomic systems have been used. The rigid rotor model of the diatomic molecules, i.e. when the distance between hydrogen atoms is fixed at some average equilibrium value, has been applied in our calculation. After this preparatory stage the astrophysical $\mathrm{H}_{2}$-cooling function can be estimated at different astrophysical conditions and temperatures.
\end{abstract}

Keywords: $\mathrm{H}_{2}$ and HD Molecules, Energy Transfer Collisions, Hydrogen Cooling in ISM.

PACS numbers: 34.20.Gj, 34.50.Ez, 95.30.Dr, 95.30.Ft

\section{Introduction}

Investigations in the fields of atom-molecular and chemical physics are always of significant importance and interest in physics research. This is especially true when the considered problems are related to atomic and molecular processes in the interstellar medium (ISM) and chemical reactions of astrophysical interest ${ }^{1}$. For instance,

\footnotetext{
${ }^{*}$ Corresponding author.
}

This is an Open Access article published by World Scientific Publishing Company. It is distributed under the terms of the Creative Commons Attribution 4.0 (CC-BY) License. Further distribution of this work is permitted, provided the original work is properly cited. 
the investigation of elastic and inelastic collisions between molecules and between molecules and atoms can provide valuable information about interactions, chemical properties and energy transfer molecular dynamics. Of a particular interest are vibrational and rotational energy transfer collisions between hydrogen molecules: $\mathrm{H}_{2}+\mathrm{H}_{2}$ and $\mathrm{HD}+\mathrm{H}_{2}$ (see Ref. ${ }^{2}$ ).

The hydrogen molecule is the simplest and most abundant molecule in the universe. This is especially pertinent in giant molecular clouds and this level of abundance is important in many areas of astrophysics. To gain a further understanding of this relationship quantitative analysis involving the rotational-vibrational excitation/de-excitation rate constants in molecular hydrogen collisions is of fundamental importance. This analysis will be critical to enhancing our understanding and ability to model the energy balance in the ISM ${ }^{3}$. Further, this energy transfer processes that involves $\mathrm{H}_{2}$ molecules controls the evolution of shock fronts as well as photo-dissociation regions (PDRs) in the interstellar medium. Also, the energy transfer process between $\mathrm{H}_{2}$ molecules as well as between $\mathrm{H}_{2}$ and other atoms/molecules is important in astrophysical processes. Specifically, these processes might include the cooling of primordial gas and shock wave-induced heating in the interstellar media. It is important to note that in order to accurately model the thermal balance and kinetics of these important systems accurate state-to-state rate constants are critical $k_{v j v^{\prime} j^{\prime}}(T)$.

Obtaining accurate measurements of quantum state resolved cross sections and their associated rate coefficients is not a trivial problem. That being said, because of the complexity of such measurements there is a need to calculate such values. To obtain accurate results using theoretical calculations requires precise potential energy surfaces and reliable dynamical treatment of the collision processes. So therefore, the $\mathrm{H}_{2}-\mathrm{H}_{2}$ system needs to be reinvestigated and the latest and most accurate interaction potentials from the following works utilized ${ }^{4,5,6,7}$. In this paper a sensitivity test is applied to the new PESs for collisions of rotationally excited $\mathrm{H}_{2}$ molecules:

$$
\mathrm{H}_{2}\left(j_{1}\right)+\mathrm{H}_{2}\left(j_{2}\right) \rightarrow \mathrm{H}_{2}\left(j_{1}^{\prime}\right)+\mathrm{H}_{2}\left(j_{2}^{\prime}\right)
$$

Additionally, in this paper we provide our preliminary results for the $\mathrm{H}+\mathrm{H}_{2}$ rotational energy transfer collision, i.e.

$$
\mathrm{H}+\mathrm{H}_{2}(j) \rightarrow \mathrm{H}+\mathrm{H}_{2}\left(j^{\prime}\right)
$$

We apply the recently published $\mathrm{H}_{3}$ (see Ref. ${ }^{8}$ ) and $\mathrm{H}_{4}$ surfaces mentioned above. In order to carry out the computation of the HD-cooling function the new results from paper ${ }^{2}$ can be used, where the potential from ${ }^{6}$ has been modified and applied. The scattering cross sections and their corresponding rate coefficients are calculated using a non reactive quantum-mechanical close-coupling approach. In the next section we will briefly outline the method. Our results are presented in Section 3 . 


\section{Quantum-Mechanical Approach}

A brief outline of the quantum-mechanical close-coupling approach used in the calculations used herein is provided in this section. Further details related to this method are presented in ${ }^{9}$. In this work the hydrogen molecules are treated as linear rigid rotors. This model has also been applied in previous works. It is normally believed that within the range of kinetic energies considered for astrophysical applications that the rotor model is adequate. Specifically in this case, the logderivative matrix can be propagated across large $R$-intermolecular distances. This is true because all experimentally observable quantum data concerning the collision can be contained in the asymptotic behavior of the functions $U_{\alpha}^{J M}(R \rightarrow \infty)$. The next step then is to solve a set of coupled second order differential equations for the unknown radial functions $U_{\alpha}^{J M}(R)$ (see Ref. ${ }^{2}$ ):

$$
\begin{array}{r}
\left(\frac{d^{2}}{d R^{2}}-\frac{L(L+1)}{R^{2}}+k_{\alpha}^{2}\right) U_{\alpha}^{J M}(R)=\sum_{\alpha^{\prime}} \int\left(\phi_{\alpha}^{J M}\left(\hat{r}_{1}, \hat{r}_{2}, \vec{R}\right)\right)^{*} \\
\times 2 M_{12} V\left(\vec{r}_{1}, \vec{r}_{2}, \vec{R}\right) \phi_{\alpha^{\prime}}^{J M}\left(\hat{r}_{1}, \hat{r}_{2}, \vec{R}\right) U_{\alpha^{\prime}}^{J M}(R) d \hat{r}_{1} d \hat{r}_{2} d \hat{R}
\end{array}
$$

where $\alpha \equiv\left(j_{1} j_{2} j_{12} L\right), j_{1}+j_{2}=j_{12}$ and $j_{12}+L=J$. We apply the hybrid modified log-derivative-Airy propagator in the general purpose scattering program MOLSCAT (see Ref. ${ }^{10}$ ) to solve the coupled radial equations (3). Different propagator schemes included in MOLSCAT have been tested. Our calculations showed that other propagators can also provide quite stable results. The numerical results are matched to the known asymptotic solution to derive the physical scattering $S$-matrix:

$$
U_{\alpha}^{J} \underset{R \rightarrow+\infty}{\sim} \delta_{\alpha \alpha^{\prime}} e^{-i\left(k_{\alpha \alpha} R-(l \pi / 2)\right)}-\left(\frac{k_{\alpha \alpha}}{k_{\alpha \alpha^{\prime}}}\right)^{\frac{1}{2}} \cdot S_{\alpha \alpha^{\prime}}^{J} e^{-i\left(k_{\alpha \alpha^{\prime}} R-\left(l^{\prime} \pi / 2\right)\right)} .
$$

The method was used for each partial wave until a converged cross section was obtained. It was verified that the results are converged with respect to the number of partial waves as well as the matching radius, $R_{\max }$, for all channels included in our calculations. The cross sections for rotational excitation and relaxation phenomena can be obtained directly from the $S$-matrix. In particular the cross sections for excitation from $j_{1} j_{2} \rightarrow j_{1}^{\prime} j_{2}^{\prime}$ summed over the final $m_{1}^{\prime} m_{2}^{\prime}$ and averaged over the initial $m_{1} m_{2}$ corresponding projections of the $\mathrm{H}_{2}$ molecules angular momenta $j_{1}$ and $j_{2}$ are given by:

$$
\sigma\left(j_{1}^{\prime}, j_{2}^{\prime} ; j_{1} j_{2}, \epsilon\right)=\frac{\pi}{\left(2 j_{1}+1\right)\left(2 j_{2}+1\right) k_{\alpha \alpha^{\prime}}} \sum_{J j_{12} j_{12}^{\prime} L L^{\prime}}(2 J+1)\left|\delta_{\alpha \alpha^{\prime}}-S_{\alpha \alpha^{\prime}}^{J}(E)\right|^{2} .
$$

The kinetic energy is $\epsilon=E-B_{1} j_{1}\left(j_{1}+1\right)-B_{2} j_{2}\left(j_{2}+1\right)$. Here $E$ is the total energy in the system, $B_{1(2)}=60.8 \mathrm{~cm}^{-1}$ are the rotation constants of the colliding $\mathrm{H}_{2}$ molecules, $J$ is total angular momenta of the 4-atomic system, $\alpha \equiv\left(j_{1} j_{2} j_{12} L\right)$, $k_{\alpha \alpha^{\prime}}=\left[2 M_{12}\left(E+E_{\alpha}-E_{\alpha^{\prime}}\right)\right]^{1 / 2}$ is the channel wavenumber and $E_{\alpha\left(\alpha^{\prime}\right)}$ are rotational channel energies. The relationship between the rate coefficient $k_{j_{1} j_{2} \rightarrow j_{1}^{\prime} j_{2}^{\prime}}(T)$ and the 
corresponding cross section $\sigma_{j_{1} j_{2} \rightarrow j_{1}^{\prime} j_{2}^{\prime}}\left(E_{k i n}\right)$ can be obtained through the following weighted average:

$$
k_{j_{1} j_{2} \rightarrow j_{1}^{\prime} j_{2}^{\prime}}(T)=\frac{8 k_{B} T}{\pi \mu} \frac{1}{\left(k_{B} T\right)^{2}} \int_{\varepsilon_{s}}^{\infty} \varepsilon d \varepsilon \sigma_{j_{1} j_{2} \rightarrow j_{1}^{\prime} j_{2}^{\prime}}(\varepsilon) e^{-\varepsilon / k_{B} T}
$$

where $\varepsilon=E-E_{j_{1}}-E_{j_{2}}$ is precollisional translational energy at the translational temperature $T, k_{B}$ is Boltzmann constant, $\mu$ is reduced mass of the moleculemolecule system, i.e. $\mu=M_{12}$, and $\varepsilon_{s}$ is the minimum kinetic energy for the levels $j_{1}$ and $j_{2}$ to become accessible.

Also, it would be useful to offer few comments about the astrophysical $\mathrm{H}_{2}$ - and HD-cooling functions, i.e., for example, about $\Lambda_{\mathrm{HD}}(T)$ in per unit of volume. In principle, the general (full) HD-cooling function should include contributions from different components, i.e. from all different atomic and molecular collisions, such as $\mathrm{HD}+\mathrm{H}, \mathrm{HD}+\mathrm{He}, \mathrm{HD}+\mathrm{H}_{2}$ and as well as other possible collisions. For example, in ${ }^{2}$ the important $\mathrm{HD}+o-/ p-\mathrm{H}_{2}$ collision has been considered at low temperatures of astrophysical interest. Now, the so called total cooling function is represented as follows:

$$
\Lambda_{\mathrm{HD}}(T)=n_{\mathrm{HD}} W_{\mathrm{HD}}(T)
$$

where $n_{\mathrm{HD}}$ is the number of density of $\mathrm{HD}$ and $W_{\mathrm{HD}}$ is the HD cooling function in units of $\left[\mathrm{erg} \times \mathrm{sec}^{-1}\right]$. From work ${ }^{11}$ it follows that the astrophysical line-cooling coefficient has the following expression:

$$
L(u \rightarrow l ; n, \tau)=\frac{n_{u} E_{u \rightarrow l} \omega_{u l}}{n n_{\nu}^{\prime}} A_{u \rightarrow l},
$$

where $E_{u l}=E_{u}-E_{l}$ is the energy of the radiated photon, $\omega_{u l}$ is the probability that the photon escapes absorption ${ }^{11}, E_{u}$ and $E_{l}$ are the energies of the upper and lower states, $n_{u}$ is the population of the upper level with $n_{u}=\exp \left(-E_{u} /\left(k_{B} T\right)\right)$, $A_{u \rightarrow l}$ are Einstein's coefficients for the $u \rightarrow l$ transition, $n$ and $n_{\nu}^{\prime}$ are the numbers of density of the participating species, for example $\mathrm{H}_{2}$ and HD. For the low densities in the case of optically thin primordial gas one has to set $\omega_{u l} \approx 1$. The line-cooling coefficient $L(u \rightarrow l ; n, \tau)$ is chosen so that the value $n n_{\nu}^{\prime} L(u \rightarrow l ; n, \tau)$ would be the cooling rate per unit of volume due to the transition $u \rightarrow l$ in an atom or molecule $\nu$ at an optical depth $\tau$ (see Ref. ${ }^{11}$ ). Therefore, it follows from Eqs. (7)-(8), if one takes $u \equiv j_{1} j_{2}$ and $l \equiv j_{1}^{\prime} j_{2}^{\prime}$ the following formula for $W_{\mathrm{HD}}(T)$ can be derived:

$$
W_{\mathrm{HD}}(T)=\frac{1}{n_{\mathrm{HD}}} \sum_{j_{1} j_{2}, j_{1}^{\prime} j_{2}^{\prime}} n_{j_{1} j_{2}}\left(E_{j_{1} j_{2}}-E_{j_{1}^{\prime} j_{2}^{\prime}}\right) \times A_{j_{1} j_{2} \rightarrow j_{1}^{\prime} j_{2}^{\prime}},
$$

where $E_{j_{1} j_{2}}>E_{j_{1}^{\prime} j_{2}^{\prime}}$ and the value of $n_{\mathrm{HD}} W_{\mathrm{HD}}(T)$ is equal to $n n_{\nu}^{\prime} L(u l ; n, \tau)$. Now, at the low density limit and taking into account the critical density concept the 
total cooling function can be computed with the use of the following formula:

$$
\Lambda_{\mathrm{HD}}(T)=\sum_{j_{1} j_{2}, j_{1}^{\prime} j_{2}^{\prime}} n_{\mathrm{HD}}\left(j_{1}\right) n_{\mathrm{H}_{2}}\left(j_{2}\right) k_{j_{1} j_{2} \rightarrow j_{1}^{\prime} j_{2}^{\prime}}(T) \times h \nu_{j_{1} j_{2} \rightarrow j_{1}^{\prime} j_{2}^{\prime}},
$$

which is in the units of $\left[\mathrm{erg} \times \mathrm{cm}^{-3} \times \mathrm{s}^{-1}\right]$. Here, $h \nu_{j_{1} j_{2} \rightarrow j_{1}^{\prime} j_{2}^{\prime}}$ is the emitted photon energy, $k_{j_{1} j_{2} \rightarrow j_{1}^{\prime} j_{2}^{\prime}}(T)$ is the thermal rate coefficient (6) corresponding to the rotational transitions $j_{1} j_{2} \rightarrow j_{1}^{\prime} j_{2}^{\prime}$. This result is the main reason that gaining knowledge about the rotational and possibly vibrational excitation/de-excitation rate constants in atomic and molecular hydrogen-hydrogen collisions, such as $\mathrm{H}_{2}+\mathrm{H}_{2}, \mathrm{HD}+\mathrm{H}_{2}$, $\mathrm{HD}+\mathrm{H}$ etc, is of importance for understanding and modeling the energy balance in the interstellar medium. Therefore, for comparison purposes, it would be useful and very interesting to carry out new computations for the rotational-vibrational integral cross sections and corresponding thermal rate coefficients for the low-energy $\mathrm{H}_{2}+\mathrm{H}$ and $\mathrm{HD}+\mathrm{H}$ collisions. In this case different $\mathrm{H}_{3}$ PESs could be applied.

\section{Computational Results}

As we mentioned in the Introduction, we apply different $\mathrm{H}_{2}-\mathrm{H}_{2} \mathrm{PESs}$ from works 4,5 and ${ }^{6,7}$. For example, the potentials ${ }^{4,7}$ have been constructed for the vibrationally averaged rigid rotor model of the $\mathrm{H}_{2}-\mathrm{H}_{2}$ system. The bond length between hydrogen atoms was fixed at 1.449 a.u. or $0.7668 \AA$. These PESs are defined by the center-ofmass intermolecular distance, $R$, and three angles: $\theta_{1}$ and $\theta_{2}$ are the plane angles and $\phi_{12}$ is the relative torsional angle. In contrast, the BMKP ${ }^{5}$ and PES from ${ }^{6}$ are global six-dimensional surfaces for two hydrogen molecules. These PESs were especially constructed to represent the whole interaction region of the chemical reaction dynamics of the four-atomic system and to provide an accurate as possible van der Waals well.

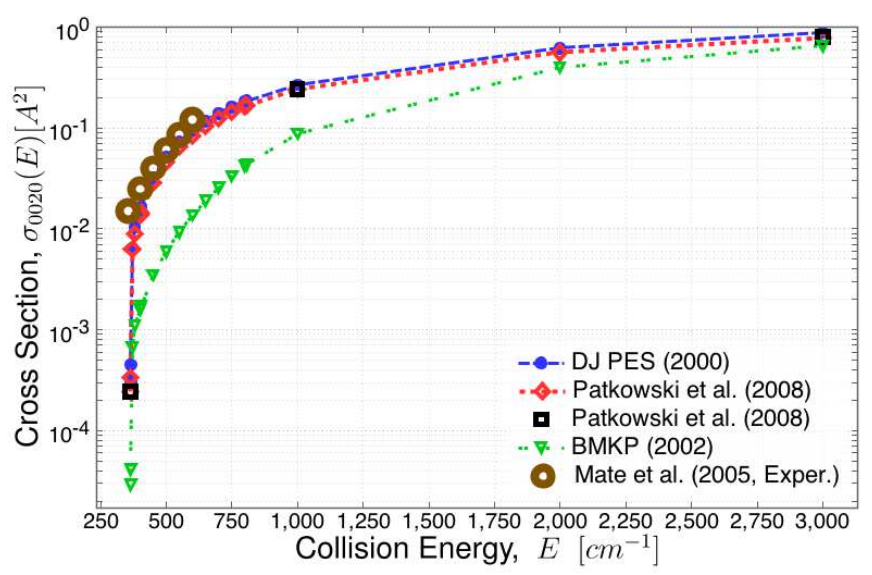

Fig. 1. The cross section of the excitation collision in $\mathrm{H}_{2}(0)+\mathrm{H}_{2}(0) \rightarrow \mathrm{H}_{2}(2)+\mathrm{H}_{2}(0)$. The lowenergy experimental data from work ${ }^{12}$ are also shown. 
The primary goal of this work was to undertake a full quantum-mechanical calculation for different rotational transitions in $\mathrm{H}_{2}+\mathrm{H}_{2}$ and $\mathrm{H}+\mathrm{H}_{2}$. These results allowed us to conduct a comparative study and evaluate the sensitivity of the PESs. Therefore, a large number of test calculations have been performed to secure the convergence of the results with respect to all parameters that enter into the propagation of the Schrödinger equation (3). This includes the intermolecular distance $R$, the total angular momentum $J$ of the four and three atomic systems, $N_{l v l}$ the number of rotational levels to be included in the close coupling expansion and others (see the MOLSCAT manual ${ }^{10}$ ). We reached convergence for the integral cross sections, $\sigma\left(E_{k i n}\right)$, in all considered collisions with the use of different PESs applied in this work. The numerical solution of the Eq. (3) have been carried out from $r_{\text {min }} \approx 1 \AA$ to $r_{\text {max }} \approx 30 \AA$. We also applied a few different propagators included in the MOLSCAT program.

In Fig. 1 one can see the computational results for the rotational excitation: $\mathrm{H}_{2}(0)+\mathrm{H}_{2}(0) \rightarrow \mathrm{H}_{2}(2)+\mathrm{H}_{2}(0)$, where the rotational quantum numbers of the molecules are shown in the brackets. For example, it is seen that the PES from work ${ }^{5}$ (the green broken line with triangles down) outputs significantly lower results for the cross section $\sigma_{0020}\left(E_{t o t}\right)$ than other potentials ${ }^{4}$ (the blue broken line with circles) and PES ${ }^{7}$ (the red broken line with diamonds). The black squares are the results computed with Patkowski et al., PES ${ }^{7}$ for a few energy points but with higher values of the MOLSCAT parameters in order to check the numerical convergence of the results. One can see, that the DJ ${ }^{4}$ and Patkowski et al., ${ }^{7}$ PESs provide a very good agreement with experimental data from work ${ }^{12}$. The temperature dependence of the rotational energy transfer thermal rate coefficients in the

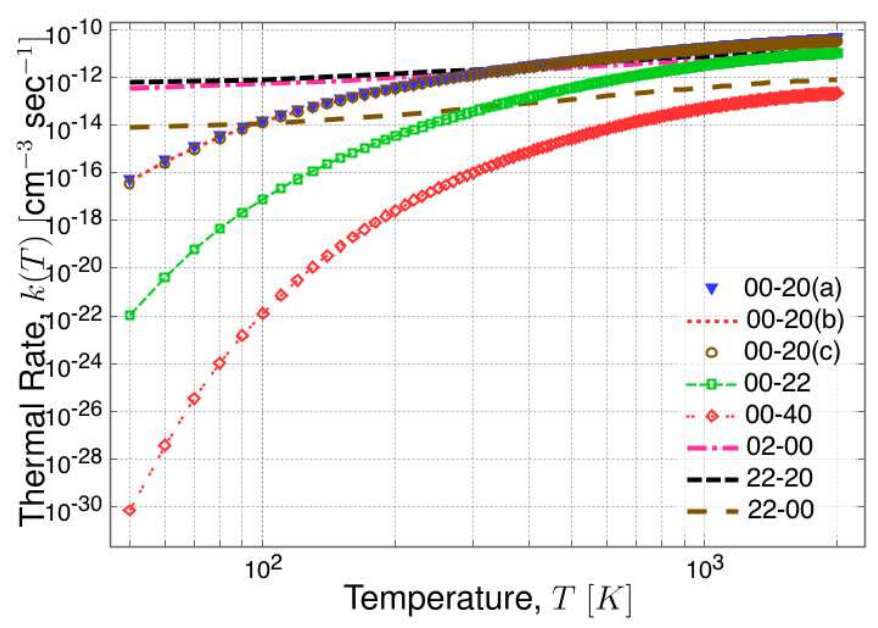

Fig. 2. The non-reactive thermal rate coefficients in $\mathrm{H}_{2}+\mathrm{H}_{2}$ : the following six channels are considered: $\mathrm{H}_{2}(0)+\mathrm{H}_{2}(0) \rightarrow \mathrm{H}_{2}(2)+\mathrm{H}_{2}(0), \mathrm{H}_{2}(0)+\mathrm{H}_{2}(0) \rightarrow \mathrm{H}_{2}(2)+\mathrm{H}_{2}(2), \mathrm{H}_{2}(0)+\mathrm{H}_{2}(0) \rightarrow \mathrm{H}_{2}(0)+\mathrm{H}_{2}(4)$, $\mathrm{H}_{2}(0)+\mathrm{H}_{2}(2) \rightarrow \mathrm{H}_{2}(0)+\mathrm{H}_{2}(0), \mathrm{H}_{2}(2)+\mathrm{H}_{2}(2) \rightarrow \mathrm{H}_{2}(2)+\mathrm{H}_{2}(0)$, and $\mathrm{H}_{2}(2)+\mathrm{H}_{2}(2) \rightarrow \mathrm{H}_{2}(0)+\mathrm{H}_{2}(0)$. 


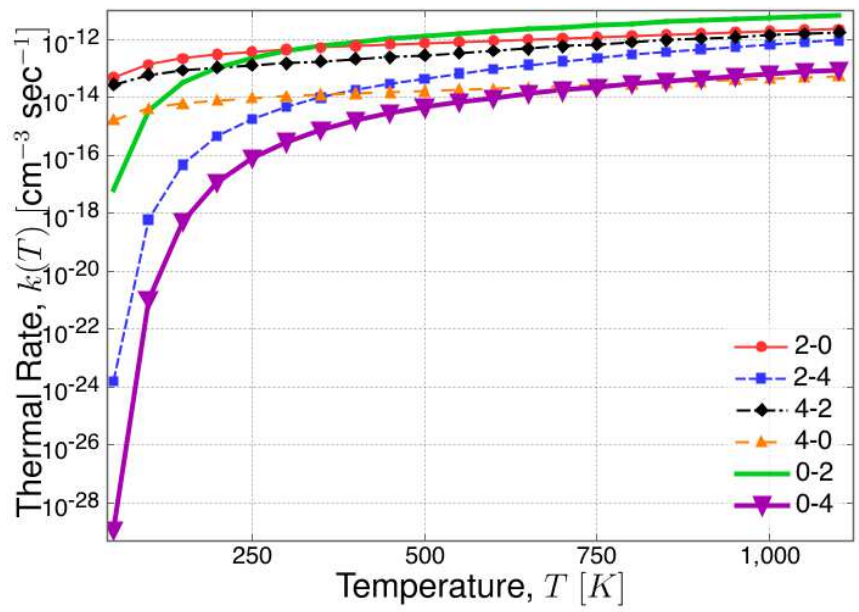

Fig. 3. The non-reactive thermal rate coefficients in $\mathrm{H}+\mathrm{H}_{2}$ : the following cases are considered: $\mathrm{H}+\mathrm{H}_{2}(0) \rightarrow \mathrm{H}+\mathrm{H}_{2}(2), \mathrm{H}+\mathrm{H}_{2}(2) \rightarrow \mathrm{H}+\mathrm{H}_{2}(0), \mathrm{H}+\mathrm{H}_{2}(2) \rightarrow \mathrm{H}+\mathrm{H}_{2}(4), \mathrm{H}+\mathrm{H}_{2}(4) \rightarrow \mathrm{H}+\mathrm{H}_{2}(2)$, $\mathrm{H}+\mathrm{H}_{2}(4) \rightarrow \mathrm{H}+\mathrm{H}_{2}(0), \mathrm{H}+\mathrm{H}_{2}(0) \rightarrow \mathrm{H}+\mathrm{H}_{2}(2), \mathrm{H}+\mathrm{H}_{2}(0) \rightarrow \mathrm{H}+\mathrm{H}_{2}(4)$.

$\mathrm{H}_{2}+\mathrm{H}_{2}$ system is represented in Fig. 2 for three different rotational excitation processes, i.e. 00-20, 00-22, 00-40 and for three de-excitation collisions: 02-00, 22-02, and 22-00. These results are obtained with the PES from ${ }^{7}$. The results 00-20 (a), (b), and (c) are obtained with the use of different MOLSCAT parameters in order to check the numerical convergence. Further these results can be directly used to estimate the contribution of (1) to the full $\mathrm{H}_{2}$ astrophysical cooling function $\Lambda_{\mathrm{H}_{2}}$. Another important but three-atomic collision (2) has also been considered in this work. Fig. 3 shows our data for few low-level rotational thermal rates in this important case. The $\mathrm{H}_{3}$ PES from ${ }^{8}$ was used. The following rotational transitions in $\mathrm{H}_{2}$ are calculated: $2 \rightarrow 0,2 \rightarrow 4,4 \rightarrow 2,4 \rightarrow 0,0 \rightarrow 2,0 \rightarrow 4$. These results can be used to perform a rough estimation of the contribution of the proces (2) to the total $\mathrm{H}_{2}$-cooling function and make a comparison, for example, with the total HD-cooling function.

In conclusion, from Eq. (10) one can see that in order to make an estimation of the cooling function (10) it is necessary, first, to compute the thermal rate coefficients for different de-excitation/excitation collisions, i.e. $k_{j_{1} j_{2} \rightarrow j_{1}^{\prime} j_{2}^{\prime}}(T)$ from Eq. (6). Therefore, comparing our previous results ${ }^{2}$ for the $\mathrm{HD}+\mathrm{H}_{2}$ rates with the current results for $\mathrm{H}_{2}+\mathrm{H}_{2}$ and $\mathrm{H}+\mathrm{H}_{2}$, one can see that at low temperatures $(T \lesssim 200 K)$ the HD-cooling function should exhibit significantly higher values than the corresponding $\mathrm{H}_{2}$ cooling function. Computations are in progress in order to obtain more expanded results for different rotational transitions in $\mathrm{H}_{2}+\mathrm{H}_{2}$ and $\mathrm{H}+\mathrm{H}_{2}$. Additionally, an estimation of the contribution of the vibrational degrees of freedom in $\mathrm{H}_{2}$ is being considered. 


\section{References}

1. A. G. G. M. Tielens, The Physics and Chemistry of the Interstellar Medium, (Cambridge University Press, Cambridge, 2005).

2. R. A. Sultanov, D. Guster, and S. K. Adhikari, J. Phys. B: At. Mol. Opt. Phys. 49 015203 (2016).

3. S. C. O. Glover and T. Abel, Mon. Not. R. Astron. Soc. 388, 1627 (2008).

4. P. Diep and J. K. Johnson, J. Chem. Phys. 1124465 (2000).

5. A. I. Boothroyd, P. G. Martin, W. J. Keogh, and M. J. Peterson, J. Chem. Phys. 116 666 (2002).

6. R. J. Hinde, J. Chem. Phys. 128, 154308 (2008).

7. K. Patkowski, W. Cencek, P. Jankowski, K. Szalewicz, J. B. Mehl, G. Garberoglio, and A. H. Harvey, J. Chem. Phys. 129, 094304 (2008).

8. A. I. Boothroyd, W. J. Keogh, P. G. Martin, and M. R. Peterson, J. Chem. Phys. 104, 7139 (1996).

9. S. Green, J. Chem. Phys. 622271 (1975).

10. J. M. Hutson, S. Green, Molscat ver. 14 (1994) (Distrib. by Collabor. Comp. Proj. 6, Daresbury Lab., UK, Eng. Phys. Sci. Res. Council).

11. D. Hollenbach and C. F. McKee, Astrophys. J. Suppl. Ser. 41555 (1979).

12. B. Mate, F. Thibault, G. Tejeda, J. M. Fernandez, and S. Montero, J. Chem. Phys. 122064313 (2005). 